\title{
A Top-Down Account of Linear Canonical Transforms ${ }^{\star}$
}

\author{
Kurt Bernardo WOLF
}

Instituto de Ciencias Físicas, Universidad Nacional Autónoma de México, Av. Universidad s/n, Cuernavaca, Mor. 62210, México

E-mail: bwolf@fis.unam.mx

URL: http: //www.fis.unam.mx/ bwolf/

Received April 24, 2012, in final form June 01, 2012; Published online June 06, 2012

http://dx.doi.org/10.3842/SIGMA.2012.033

\begin{abstract}
We contend that what are called Linear Canonical Transforms (LCTs) should be seen as a part of the theory of unitary irreducible representations of the ' $2+1$ ' Lorentz group. The integral kernel representation found by Collins, Moshinsky and Quesne, and the radial and hyperbolic LCTs introduced thereafter, belong to the discrete and continuous representation series of the Lorentz group in its parabolic subgroup reduction. The reduction by the elliptic and hyperbolic subgroups can also be considered to yield LCTs that act on functions, discrete or continuous in other Hilbert spaces. We gather the summation and integration kernels reported by Basu and Wolf when studiying all discrete, continuous, and mixed representations of the linear group of $2 \times 2$ real matrices. We add some comments on why all should be considered canonical.
\end{abstract}

Key words: linear transforms; canonical transforms; Lie group $\mathrm{Sp}(2, \mathrm{R})$

2010 Mathematics Subject Classification: 20C10; 20C35; 33C15; 33C45

\section{Introduction}

Linear canonical transforms (LCTs) have been developed as the diffraction integral kernel for generic paraxial optical systems by Stuart A. Collins [7], and also defined as the group of unitary integral transforms that preserves the basic Heisenberg uncertainty relation of quantum mechanics in $D=1$ or higher dimensions by Marcos Moshinsky and Christiane Quesne $[16,17,23]$. Further, LCTs can be seen as the group actions generated by the Lie algebra of quadratic Hamiltonian operators [25]. Also, radial [18, 26] and hyperbolic [27] canonical transforms have been defined after separation of variables in $D \geq 2$ dimensions. More recently there has been interest in canonical transformations that are represented by matrices, infinite, semiinfinite or finite-dimensional - the latter due to the finite capacity of measuring and storing devices, but with serious concomitant difficulties.

In both the optical Lagrangian formulation [7] or in the quantum-mechanical Hamiltonian approach [16], one arrives at a parametrization of all possible linear transformations through a $2 D \times 2 D$ real symplectic matrix $\mathbf{M} \in \mathrm{Sp}(2 D, \mathrm{R})$. For reference we gather here the basic $D=1$-dimensional formulas. We consider the matrix

$$
\mathbf{M}=\left(\begin{array}{ll}
a & b \\
c & d
\end{array}\right), \quad a d-b c=1,
$$

as the presentation of the group $\operatorname{Sp}(2, \mathrm{R})$, which is equal to the groups of all real $2 \times 2$ matrices of unit determinant $\mathrm{SL}(2, \mathrm{R})$, and is isomorphic through a complex similarity transformation to the group $\mathrm{SU}(1,1)$ of $2 \times 2$ pseudo-unitary matrices. This group of $2 \times 2$ matrices in fact covers

\footnotetext{
*This paper is a contribution to the Special Issue "Superintegrability, Exact Solvability, and Special Functions". The full collection is available at http://www.emis.de/journals/SIGMA/SESSF2012.html
} 
twice, i.e., is 2:1 homomorphic to the ' $2+1$ '-Lorentz group of $3 \times 3$ pseudo-orthogonal matrices $\mathbf{L}( \pm \mathbf{M}) \in \mathrm{SO}(2,1)$

$$
\left(\begin{array}{ll}
a & b \\
c & d
\end{array}\right) \stackrel{2: 1}{\longleftrightarrow}\left(\begin{array}{ccc}
\frac{1}{2}\left(a^{2}-b^{2}-c^{2}+d^{2}\right) & b d-a c & \frac{1}{2}\left(a^{2}-b^{2}+c^{2}-d^{2}\right) \\
c d-a b & a d+b c & -c d-a b \\
\frac{1}{2}\left(a^{2}+b^{2}-c^{2}-d^{2}\right) & -b d-a c & \frac{1}{2}\left(a^{2}+b^{2}+c^{2}+d^{2}\right)
\end{array}\right) .
$$

These accidental iso- and homomorphisms between symplectic and relativity groups have been exploited to describe and relate various physical and optical models [11, 15, 19, 31].

However, in spite of its apparent simplicity, the group of $2 \times 2$ matrices (1) has an infinite cover group $\overline{\mathrm{Sp}}(2, \mathrm{R})$. It is the double cover - the metaplectic group $\mathrm{Mp}(2, \mathrm{R})$ - which is represented by the well-known integral form of linear canonical transforms $\mathcal{C}_{\mathrm{M}} \equiv \mathcal{C}\left(\begin{array}{ll}a & b \\ c & d\end{array}\right)$ of the usual Hilbert space of functions $f(x) \in \mathcal{L}^{2}(\mathrm{R})$,

$$
\begin{aligned}
& f_{\mathrm{M}}(x) \equiv\left(\mathcal{C}_{\mathrm{M}} f\right)(x)=\int_{\mathrm{R}} \mathrm{d} x^{\prime} C_{\mathrm{M}}\left(x, x^{\prime}\right) f\left(x^{\prime}\right), \\
& C_{\mathrm{M}}\left(x, x^{\prime}\right):=\frac{1}{\sqrt{2 \pi \mathrm{i} b}} \exp \left(\frac{\mathrm{i}}{2 b}\left(d x^{2}-2 x x^{\prime}+a x^{\prime 2}\right)\right),
\end{aligned}
$$

where the phase of the prefactor is taken to be

$$
\frac{1}{\sqrt{2 \pi \mathrm{i} b}}=\frac{1}{\sqrt{2 \pi|b|}} \exp \left(-\mathrm{i} \frac{1}{4} \pi \operatorname{sign} b\right) \text {. }
$$

Unitarity is evident in that $C_{\mathrm{M}^{-1}}\left(x, x^{\prime}\right)=C_{\mathrm{M}}\left(x^{\prime}, x\right)^{*}$.

Phases are quite delicate here; Collins did not consider the group property of the transforms [7]; yet Moshinsky and Quesne [16] realized that $\mathcal{C}_{\mathrm{M}_{1}} \mathcal{C}_{\mathrm{M}_{2}}= \pm \mathcal{C}_{\mathrm{M}_{1} \mathrm{M}_{2}}$, with a sign depending on the signs of the $b_{1}, b_{2}$ and $b_{12}$ elements in a rather complicated way [29, Chapter 9]. The double-cover issue can be simplified by observing that for $\mathbf{F}=\left(\begin{array}{cc}0 & 1 \\ -1 & 0\end{array}\right)$, the kernel (3) is that of the Fourier transform $\mathcal{F}$, but for a phase: $\mathcal{C}_{\mathrm{F}}=e^{-\mathrm{i} \pi / 4} \mathcal{F}$. Since $\mathcal{F}^{4}=1$, only $\mathcal{C}_{\mathrm{F}}^{8}$ will return the cycle to the unit 1 . In the limit $b \rightarrow 0$ from the lower complex half-plane, the kernel (2) becomes a Dirac delta,

$$
\lim _{b \rightarrow 0} C_{\mathrm{M}}\left(x, x^{\prime}\right)=\frac{\exp \left(\mathrm{i} c x^{2} / 2 a\right)}{\sqrt{a}} \delta\left(x^{\prime}-\frac{x}{a}\right) .
$$

We may skip further detailed consideration of the $D=1$ integral linear canonical transforms and their properties, which are mostly standard knowledge, and from whose $D=2$ case one can build the radial and hyperbolic LCTs in a bottom-up construction. Rather, the purpose of this review is to give a top-down panorama of LCTs.

In Section 2 we return to the Lie algebra so $(2,1)$ of the Lorentz group $\mathrm{SO}(2,1)$ realized by second-order differential operators corresponding to harmonic and repusive oscillators, with a singular centrifugal or centripetal potential, and the generator of scaling. Linear combinations yield free propagation and a square-radius coordinate. In Section 3 we list the eigenfunctions and spectra of those operators for the centrifugal case that fall into the Bargmann $\mathcal{D}_{k}$ discrete series of representations. There are three subgroup orbits that are examined in three subsections: elliptic, parabolic, and hyperbolic. Section 4 follows the same structure for the centripetal case that fall into the Bargmann $\mathcal{C}_{s}^{\varepsilon}$ continuous series. This is what we deem to be six faces that $\mathrm{Sp}(2, \mathrm{R})$ linear canonical transforms can show in various Hilbert spaces. The resulting matrix and integral kernels were computed in 1981 by Basu and Wolf [6], so the results are not new, but are gathered here for the first time as proper, unitary LCTs. The concluding Section 5 offers some reasons to call all representations canonical, and some comments on finite-dimensional approximations to these transforms. 


\section{The Lorentz algebra}

The generic form of the second-order differential operator realization of the Lorentz Lie algebra so $(2,1)$ can be obtained from the oscillator algebra [17], adding a term $\gamma / r^{2}$ to the second derivative, whose interpretation is that of a centrifugal potential as $\gamma=\bar{m}^{2}-\frac{1}{4} \geq-\frac{1}{4}$ for solutions of angular momentum $\bar{m} \in$ Z. Separation into hyperbolic coordinates yields $\gamma<-\frac{1}{4}$ when added by a centripetal force. The formal operators are associated with Hamiltonians, and are generators of $\mathrm{Sp}(2, \mathrm{R})$ subgroups as follows:

$$
\begin{array}{rlrl}
J_{0}^{\gamma}:=\frac{1}{4}\left(-\frac{\mathrm{d}^{2}}{\mathrm{~d} r^{2}}+\frac{\gamma}{r^{2}}+r^{2}\right), & \exp \left(\mathrm{i} \phi J_{0}^{\gamma}\right) & \mapsto\left(\begin{array}{cc}
\cos \frac{1}{2} \phi & -\sin \frac{1}{2} \phi \\
\sin \frac{1}{2} \phi & \cos \frac{1}{2} \phi
\end{array}\right), \\
J_{1}^{\gamma}:=\frac{1}{4}\left(-\frac{\mathrm{d}^{2}}{\mathrm{~d} r^{2}}+\frac{\gamma}{r^{2}}-r^{2}\right), & \exp \left(\mathrm{i} \zeta J_{1}^{\gamma}\right) \mapsto\left(\begin{array}{rr}
\cosh \frac{1}{2} \zeta & -\sinh \frac{1}{2} \zeta \\
-\sinh \frac{1}{2} \zeta & \cosh \frac{1}{2} \zeta
\end{array}\right), \\
J_{2}^{\gamma}:=-\frac{\mathrm{i}}{2}\left(r \frac{\mathrm{d}}{\mathrm{d} r}+\frac{1}{2}\right), & \exp \left(\mathrm{i} \alpha J_{2}^{\gamma}\right) \mapsto\left(\begin{array}{cc}
\exp -\frac{1}{2} \alpha & 0 \\
0 & \exp +\frac{1}{2} \alpha
\end{array}\right),
\end{array}
$$

where the 'harmonic oscillator' $J_{0}^{\gamma}$ generates the compact (elliptic) SO(2) subgroup (and its covers), while the 'repulsive oscillator' $J_{1}^{\gamma}$ and 'scaling operator' $J_{2}^{\gamma}$ generate equivalent noncompact (hyperbolic) subgroups $\mathrm{SO}(1,1)$. Their commutation relations are

$$
\left[J_{1}^{\gamma}, J_{2}^{\gamma}\right]=-\mathrm{i} J_{0}^{\gamma}, \quad\left[J_{2}^{\gamma}, J_{0}^{\gamma}\right]=\mathrm{i} J_{1}^{\gamma}, \quad\left[J_{0}^{\gamma}, J_{1}^{\gamma}\right]=\mathrm{i} J_{2}^{\gamma} .
$$

Also relevant are the linear combinations

$$
\begin{array}{lll}
J_{+}^{\gamma}:=J_{0}^{\gamma}+J_{1}^{\gamma}=\frac{1}{2}\left(-\frac{\mathrm{d}^{2}}{\mathrm{~d} r^{2}}+\frac{\gamma}{r^{2}}\right), & \exp \left(\mathrm{i} b J_{+}^{\gamma}\right) \mapsto\left(\begin{array}{cc}
1 & -b \\
0 & 1
\end{array}\right), \\
J_{-}^{\gamma}:=J_{0}^{\gamma}-J_{1}^{\gamma}=\frac{1}{2} r^{2}, & \exp \left(\mathrm{i} c J_{-}^{\gamma}\right) \mapsto\left(\begin{array}{cc}
1 & 0 \\
c & 1
\end{array}\right),
\end{array}
$$

where the 'free system' $J_{+}^{\gamma}$ and the 'square-radius' $J_{-}^{\gamma}$ generate the Euclidean (parabolic) subgroups ISO(1), which are equivalent under the Fourier transform. Note carefully that these are not 'raising and lowering' operators for discrete eigenvector bases. Instead and particularly, $J_{-}^{\gamma}$ determines the diagonal radial position operator with respect to which we refer the eigenfunctions of all other operators, as done below. These eigenfunctions depend crucially on the value of $\gamma$ in (5), as given by the Casimir eigenvalue

$$
\begin{aligned}
& C:=\left(J_{1}^{\gamma}\right)^{2}+\left(J_{2}^{\gamma}\right)^{2}-\left(J_{0}^{\gamma}\right)^{2}=\kappa 1, \\
& \kappa=-\frac{1}{4} \gamma+\frac{3}{16}=: k(1-k), \quad k=\frac{1}{2}\left(1 \pm \sqrt{\gamma+\frac{1}{4}}\right),
\end{aligned}
$$

where $k$ is the Bargmann index [2] that determines (up to parities) the essentially self-adjoint irreducible representations of the algebra in a Hilbert space $\mathcal{L}^{2}\left(\mathrm{R}^{+}\right)$with measure $\mathrm{d} r$ - which are unexpectedly imbricate.

\section{The $\mathcal{D}_{k}$ canonical transforms}

We consider first the case when the coefficient $\gamma$ is of centrifugal origin in $D=2$ using polar coordinates $x=r \cos \theta$ and $y=r \sin \theta, r \in \mathrm{R}^{+}, \theta$ modulo $2 \pi$. We want the measure to be $\mathrm{d} r$, so after similarity transformation, $\sqrt{r} \nabla^{2} / \sqrt{r}=\partial_{r}^{2}+r^{-2}\left(\frac{1}{4}+\partial_{\theta}^{2}\right)$, with $\partial_{\theta}^{2} \mapsto-\bar{m}^{2}$ for the subspaces of angular momentum $\bar{m} \in \mathrm{Z}$ so that $k=\frac{1}{2}(|\bar{m}|+1) \in\left\{\frac{1}{2}, 1, \frac{3}{2}, \ldots\right\}$. This is the Bargmann 
discrete representation series $\mathcal{D}_{k}^{ \pm}[2] .^{1}$ The eigenfunctions and spectra of the five operators (5) and (7), are [6]

$$
\begin{aligned}
{ }^{0} \Phi_{m}^{k}(r) & =\sqrt{\frac{2 n !}{(2 k-n-1) !}} r^{2 k-1 / 2} e^{-r^{2} / 2} L_{n}^{(2 k-1)}\left(r^{2}\right) \\
& =\frac{\sqrt{2(2 k+n-1) !}}{\Gamma(2 k) \sqrt{n !}} r^{2 k-1 / 2} e^{-r^{2} / 2}{ }_{1} F_{1}\left(\begin{array}{c}
-n \\
2 k
\end{array} ; r^{2}\right), \quad m=k+n, \quad n \in \mathrm{Z}, \\
{ }^{+} \Phi_{\rho}^{k}(r) & =e^{\mathrm{i} \pi k \sqrt{\rho r} J_{2 k-1}(\rho r), \quad \rho \in \mathrm{R}^{+},} \\
{ }^{-} \Phi_{\rho}^{k}(r) & =\delta(\rho-r), \quad \rho \in \mathrm{R}^{+}, \\
{ }^{1} \Phi_{\mu}^{k}(r) & =\frac{e^{\mathrm{i} \pi(2 k+\mu) / 2} 2^{\mathrm{i} \mu} \Gamma(k+\mathrm{i} \mu)}{\Gamma(2 k) \sqrt{\pi}} \frac{1}{\sqrt{r}} M_{\mathrm{i} \mu, k-1 / 2}\left(-\mathrm{i} r^{2}\right), \\
& =\frac{e^{\mathrm{i} \pi(k+\mu) / 2} 2^{\mathrm{i} \mu} \Gamma(k+\mathrm{i} \mu)}{\Gamma(2 k) \sqrt{\pi}} r^{2 k-1 / 2} e^{\mathrm{i} r^{2} / 2}{ }_{1} F_{1}\left(\begin{array}{c}
k-\mathrm{i} \mu \\
2 k
\end{array} ;-\mathrm{i} r^{2}\right), \quad \mu \in \mathrm{R}, \\
{ }^{2} \Phi_{\mu}^{k}(r) & =\frac{1}{\sqrt{\pi}} r^{-1 / 2+\mathrm{i} \mu}, \quad \mu \in \mathrm{R},
\end{aligned}
$$

where $L_{n}^{(\mu)}$ are the Laguerre polynomials, $J_{\mu}$ is the Bessel function of the first kind, and $M_{\lambda, \mu}$ is one of the Whittaker functions. In Dirac notation, ${ }^{X} \Phi_{\lambda}^{k}(r) \equiv{ }_{-}\langle k, r \mid k, \lambda\rangle_{X}$, with $X \in$ $\{0,1,2,+,-\}$ indicating the eigenkets of $J_{X}^{\gamma}$, with eigenvalues $\lambda \in \Sigma_{X}$ in the spectra of (9).

Next, we should obtain the transformation of the functions in (9) under the generic LCT $\mathcal{C}_{\mathrm{M}} \equiv \mathcal{C}\left(\begin{array}{ll}a & b \\ c & d\end{array}\right)$. To this end for each generator $J_{X}^{\gamma}$ in the lists (5) and (7) we decompose $\mathcal{C}_{\mathrm{M}}$ into a right-ordered product of the subgroup with a diagonal-plus-phase transformation as (4),

$$
\mathcal{C}\left(\begin{array}{ll}
a & b \\
c & d
\end{array}\right)=\mathcal{C}\left(\begin{array}{cc}
a^{\prime} & 0 \\
c^{\prime} & 1 / a^{\prime}
\end{array}\right) \exp \left(\mathrm{i} \alpha J_{X}^{\gamma}\right)
$$

where $a^{\prime}, c^{\prime}$ and $\alpha$ will be algebraic and trigonometric functions of $a, b, c, d$, respecting $a d-b c=1$. The right factor applied to ${ }^{X} \Phi_{\lambda}^{k}(r)$ will multiply it by the phase $e^{\mathrm{i} \lambda \alpha}$, while the left factor will be given by (4), with the sole precaution of taking $1 / \sqrt{a} \equiv(\operatorname{sign} a)^{2 k} / \sqrt{|a|}$ stemming from the radial reduction of the $D=2$ case with angular momentum.

\subsection{Elliptic basis: lower-bound discrete $\mathcal{D}_{k}$-LCTs}

We consider first the eigenbasis of the compact generator $J_{0}^{\gamma},{ }^{0} \Phi_{m}^{k}(r)$, in the $\mathcal{D}_{k}^{+}$Bargmann representation series, whose eigenvalues are lower-bound and discrete, $\lambda \equiv m=k+n, n \in \mathrm{Z}_{0}^{+}$. The decomposition (10) yields $a^{\prime}=\sqrt{a^{2}+b^{2}}, a^{\prime} c^{\prime}=a c+b d$ and $e^{\mathrm{i} \alpha}=(a-\mathrm{i} b) /(a+\mathrm{i} b)$. Then,

$$
\mathcal{C}\left(\begin{array}{ll}
a & b \\
c & d
\end{array}\right){ }^{0} \Phi_{m}^{k}(r)=\left(\frac{a-\mathrm{i} b}{a+\mathrm{i} b}\right)^{m} \frac{\exp \left(\mathrm{i}^{2} \frac{a c+b d}{2\left(a^{2}+b^{2}\right)}\right)}{\left(a^{2}+b^{2}\right)^{1 / 4}} \Phi_{m}^{k}\left(\frac{r}{\sqrt{a^{2}+b^{2}}}\right) .
$$

From this we can find the representation matrices of the group,

$$
X_{D_{\lambda, \lambda^{\prime}}^{k}}(\mathbf{M})=\int_{0}^{\infty}{ }_{X}\langle k, \lambda \mid k, r\rangle_{-} \mathrm{d} r_{-}\left\langle k, r\left|\mathcal{C}_{\mathbf{M}}\right| k, \lambda^{\prime}\right\rangle_{X}
$$

\footnotetext{
${ }^{1}$ The same were named by I.M. Gel'fand and M.A. Naŭmark to be the complementary representation series $[8,21]$. Note that $k \leftrightarrow 1-k$ correspond to the same $\kappa$; the interval $0<k<1$ is exceptional in that the operators have a one-parameter family of self-adjoint extensions [30], and also harbor the exceptional, or supplementary irreducible representation series $[2,8,21]$. We shall be working within the Friedrichs extension.
} 
by straightforward integration of the confluent hypergeometric functions that appear in the tables of Gradshteln and Ryzhik [10]. In the $\mathrm{SO}(2)$ basis, this is a matrix with rows and columns numbered by $m=k+n, n \in \mathrm{Z}_{0}^{+}$, that was found by Bargmann [2],

$$
\begin{aligned}
{ }^{0} D_{m, m^{\prime}}^{k}\left(\begin{array}{ll}
a & b \\
c & d
\end{array}\right):= & \left({ }^{0} \Phi_{m}^{k}, \mathcal{C}\left(\begin{array}{ll}
a & b \\
c & d
\end{array}\right){ }^{0} \Phi_{m^{\prime}}^{k}\right)=\int_{0}^{\infty} \mathrm{d} r^{0} \Phi_{m}^{k}(r)^{*} \mathcal{C}\left(\begin{array}{ll}
a & b \\
c & d
\end{array}\right){ }^{0} \Phi_{m^{\prime}}^{k}(r) \\
= & \frac{2^{2 k} \Gamma\left(m+m^{\prime}\right)}{\sqrt{\Gamma(k+m) \Gamma(1-k+m) \Gamma\left(k+m^{\prime}\right) \Gamma\left(1-k+m^{\prime}\right)}} \\
& \times[(d-a)-\mathrm{i}(b+c)]^{m-k}[(a-d)-\mathrm{i}(b+c)]^{m^{\prime}-k}[(a+d)+\mathrm{i}(b-c)]^{-m-m^{\prime}} \\
& \times{ }_{2} F_{1}\left(\begin{array}{c}
k-m, k-m^{\prime} \\
1-m-m^{\prime}
\end{array} ; \frac{a^{2}+b^{2}+c^{2}+d^{2}+2}{a^{2}+b^{2}+c^{2}+d^{2}-2}\right) .
\end{aligned}
$$

Then, when $\mathbf{f} \equiv\left\{f_{n}\right\}_{n=0}^{\infty}$ is a vector in the Hilbert space of square-summable sequences $\ell^{2}\left(\mathrm{Z}_{0}^{+}\right)$, the $\operatorname{Sp}(2, \mathrm{R})$ action

$$
\mathbf{f}_{\mathrm{M}} \equiv \mathcal{C}_{\mathrm{M}}: \mathbf{f}={ }^{0} \mathbf{D}^{k} \mathbf{f}, \quad f_{\mathrm{M} ; n} \equiv\left(\mathbf{f}_{\mathrm{M}}\right)_{n}=\sum_{n^{\prime}=0}^{\infty} D_{k+n, k+n^{\prime}}^{k}(\mathbf{M}) f_{n^{\prime}}
$$

is an LCT which is unitary in the Hilbert space of sequences $\ell^{2}\left(\mathrm{Z}_{0}^{+}\right)$.

In fact, (13) provides a unitary summation transform kernel for every value $k>0$, not necessarily stemming from integer angular momentum $\bar{m}$, where $k=\frac{1}{2}(|\bar{m}|+1) \in\left\{\frac{1}{2}, 1, \frac{3}{2}, \ldots\right\}$ are single- and double-covers of the $\mathrm{SO}(2,1)$ group, i.e., single covers of $\mathrm{Sp}(2, \mathrm{R})$. When $k$ is quarter-integer (in particular $k=\frac{1}{4}$ and $\frac{3}{4}$, to be revisited below), we have representations of the metaplectic group $\operatorname{Mp}(2, \mathrm{R})$, and various higher covers for fractional $k$ 's.

In the $\mathcal{D}_{k}^{+}$Bargmann representations, the generator $J_{0}^{\gamma}$ has an equally-spaced spectrum $\{m\}_{m=k}^{\infty}$ which is bound from below by $k>0$. There is also a paired series of representations $\mathcal{D}_{k}^{-}$ where that spectrum is upper-bound by $-k$, i.e., $m=-k-n, n \in \mathrm{Z}_{0}^{+}$. This stems from the outer algebra automorphism $J_{0}^{\gamma} \leftrightarrow-J_{0}^{\gamma}$ (reversing the sign of the spectrum), $J_{1}^{\gamma} \leftrightarrow-J_{1}^{\gamma}, J_{2}^{\gamma} \leftrightarrow J_{2}^{\gamma}$, and so $J_{ \pm}^{\gamma} \leftrightarrow-J_{ \pm}^{\gamma}$. This generates an outer group automorphism whose representations in any subgroup reduction yields the $\mathcal{D}_{k}^{-}$matrices or integral kernels

$$
X_{D_{\lambda, \lambda^{\prime}}^{k,(-)}}^{k}\left(\begin{array}{ll}
a & b \\
c & d
\end{array}\right) \leftrightarrow X^{X} D_{\sigma \lambda, \sigma \lambda^{\prime}}^{k,(+)}\left(\begin{array}{cc}
a & -b \\
-c & d
\end{array}\right),
$$

where $J_{X}^{\gamma}$ has spectrum $\{\lambda\} \in \Sigma_{X}$ and parity $\sigma \in\{+,-\}$ under this automorphism. In fact, the matrices in (15) are related by a similarity transformation with $\left(\begin{array}{cc}1 & 0 \\ 0 & -1\end{array}\right)$, a non-symplectic matrix which describes reflection in geometric optics [28, Chapter 4].

We have spoken of $\mathcal{D}_{k}^{ \pm}$for $k>0$. What about $k<0$ ? The Bargmann index $k$ acts as a lower bound for the values of $m$ in $\mathcal{D}_{k}^{+}$, and as an upper bound in $\mathcal{D}_{k}^{-}$in the one-step recursion relations obtained with raising and lowering operators on the eigenvectors of $J_{0}^{\gamma}$; but they are one-way barriers. At $k=0$ the $m= \pm k$ lines cross, so that when $k<0$ is integer or halfinteger, the $m$ 's have a lower bound at negative $k$ and an upper bound at positive $-k$. Raising and lowering the $m$ 's in this range will yield a $(2 k+1)$-dimensional matrix, which is a faithful, although non-unitary irreducible representation of $\operatorname{Sp}(2, \mathrm{R})$. Of course, a well-known theorem (see [9]) states that non-compact groups do not possess finite-dimensional unitary irreducible representations.

\subsection{Parabolic basis: continuous radial $\mathcal{D}_{k}$-LCTs}

The eigenfunctions ${ }^{+} \Phi_{\rho}^{k}(r)$ and ${ }^{-} \Phi_{\rho}^{k}(r)$ in (9) of the parabolic generators $J_{+}^{\gamma}$ and $J_{-}^{\gamma}$ in (7) form generalized bases for $\mathcal{L}^{2}\left(\mathrm{R}^{+}\right)$. Consider thus the $\mathrm{Sp}(2, \mathrm{R})$ representation given as an integral 
kernel by

$$
\begin{aligned}
& { }^{-} D_{\rho, \rho^{\prime}}^{k}\left(\begin{array}{ll}
a & b \\
c & d
\end{array}\right):=\left({ }^{-} \Phi_{\rho}^{k}, \mathcal{C}\left(\begin{array}{ll}
a & b \\
c & d
\end{array}\right){ }^{-} \Phi_{\rho^{\prime}}^{k}\right)=\left({ }^{-} \Phi_{\rho}^{k}, \mathcal{C}\left(\begin{array}{ll}
a & b \\
c & d
\end{array}\right) \mathcal{C}\left(\begin{array}{cc}
0 & -1 \\
1 & 0
\end{array}\right){ }^{+} \Phi_{\rho^{\prime}}^{k}\right) \\
& =\left(-\Phi_{\rho}^{k}, \mathcal{C}\left(\begin{array}{cc}
b & 0 \\
d & 1 / b
\end{array}\right) \mathcal{C}\left(\begin{array}{cc}
1 & -a / b \\
0 & 1
\end{array}\right)+{ }^{+} \Phi_{\rho^{\prime}}^{k}\right) \\
& =\frac{e^{-\mathrm{i} \pi k}}{b} \sqrt{\rho \rho^{\prime}} \exp \left(\mathrm{i} \frac{d \rho^{2}+a \rho^{\prime 2}}{2 b}\right) J_{2 k-1}\left(\frac{\rho \rho^{\prime}}{b}\right) \\
& =\frac{2\left(\rho \rho^{\prime}\right)^{2 k-1 / 2}}{(2 \mathrm{i} b)^{2 k} \Gamma(2 k)} \exp \left(\mathrm{i} \frac{d \rho^{2}-2 \rho \rho^{\prime}+a \rho^{\prime 2}}{2 b}\right){ }_{1} F_{1}\left(\begin{array}{c}
2 k-\frac{1}{2} \\
4 k-1
\end{array} ; \frac{2 \mathrm{i} \rho \rho^{\prime}}{b}\right) \text {. }
\end{aligned}
$$

This we recognize as the radial canonical transform kernel $[18,26]$ for angular momentum $k=$ $\frac{1}{2}(\bar{m}+1)$, which can be extended to $k>0$, that acts unitarily on the functions $\mathbf{f} \equiv\{f(r)\}_{r \in \mathrm{R}^{+}}$ in the Hilbert space $\mathcal{L}^{2}\left(\mathrm{R}^{+}\right)$with measure $\mathrm{d} r$. As we had in (14), now

$$
\mathbf{f}_{\mathrm{M}} \equiv \mathcal{C}_{\mathrm{M}}: \mathbf{f}={ }^{-} \mathbf{D}^{k}(\mathbf{M}) \mathbf{f}, \quad f_{\mathrm{M}}(r) \equiv\left(\mathbf{f}_{\mathrm{M}}\right)(r)=\int_{0}^{\infty} \mathrm{d} r^{\prime} D_{r, r^{\prime}}^{k}(\mathbf{M}) f\left(r^{\prime}\right) .
$$

We regain the classical $D=1 \mathrm{LCT}$ kernel in (3) as the direct sum of the representations $k=\frac{1}{4}$ and $\frac{3}{4}$, for functions $f(x)$ whose domain is extended to $x \in \mathrm{R}$ through writing them as the sum $f(x)=f_{e}^{(1 / 4)}(x)+f_{o}^{(3 / 4)}(x)$ with even and odd parity summands $f_{e}(-r):=f_{e}(r)$ and $f_{o}(-r):=-f_{o}(r)$ respectively. For $2 k-1=\mp 1$, the integral kernels contain $J_{-1 / 2}(z)=$ $\sqrt{2 / \pi z} \cos z$ and $J_{+1 / 2}(z)=\sqrt{2 / \pi z} \sin z, z=\rho \rho^{\prime} / b$, with phases $\mathrm{i}^{2 k}=e^{\mathrm{i} \pi / 4}$ and $\mathrm{i} e^{\mathrm{i} \pi / 4}$. Their sum thus yields $e^{\mathrm{i} \pi / 4} e^{-\mathrm{i} x x^{\prime} / b}$ in the oscillating Gaussian of the original LCT kernel (3),

$$
C_{\mathrm{M}}\left(x, x^{\prime}\right)=D_{\mathrm{M}}^{(1 / 4)}\left(r, r^{\prime}\right)+D_{\mathrm{M}}^{(3 / 4)}\left(r, r^{\prime}\right) .
$$

Had we chosen the eigenbasis of the free Hamiltonian $J_{+}^{\gamma}$ instead of the square-position $J_{-}^{\gamma}$, the Bessel function ${ }^{+} \Phi_{\rho}^{k}(r)$ in (9) we would have the Hankel transform of (18) by $\mathbf{F}=\left(\begin{array}{cc}0 & 1 \\ -1 & 0\end{array}\right)$, so

$$
{ }^{+} D_{\rho, \rho^{\prime}}^{k}\left(\begin{array}{ll}
a & b \\
c & d
\end{array}\right):=\left({ }^{+} \Phi_{\rho}^{k}, \mathcal{C}\left(\begin{array}{ll}
a & b \\
c & d
\end{array}\right){ }^{+} \Phi_{\rho^{\prime}}^{k}\right)={ }^{-} D_{\rho, \rho^{\prime}}^{k}\left(\begin{array}{cc}
d & -c \\
-b & a
\end{array}\right)
$$

\subsection{Hyperbolic basis: the face of $\mathcal{D}_{k}$-LCTs}

There remain the eigenbases of the two equivalent noncompact operators in the list (5): the repulsive oscillator Hamiltonian $J_{1}^{\gamma}$ and the scaling generator $J_{2}^{\gamma}$. The latter is the simpler of the two because its eigenfunctions, ${ }^{2} \Phi_{\mu}^{k}(r)$ in (9), are the Mellin transform kernel with $\mu \in \mathrm{R}$ and $k>0$. Since we know the LCT action $\mathcal{C}_{\mathrm{M}}$ in the parabolic basis, (16)-(19), we apply it to these eigenfunctions,

$$
\begin{aligned}
\left(\mathcal{C}_{\mathrm{M}}:{ }^{2} \Phi_{\mu}^{k}\right)(r) & =\frac{1}{\sqrt{\pi}} \int_{0}^{\infty} \mathrm{d} r^{\prime} D_{r, r^{\prime}}^{k}\left(\begin{array}{ll}
a & b \\
c & d
\end{array}\right) r^{\prime-1 / 2+\mathrm{i} \mu} \\
& =\frac{e^{-\mathrm{i} \pi k}}{2^{k-\mathrm{i} \mu} \sqrt{\pi}} \frac{\Gamma(k+\mathrm{i} \mu)}{\Gamma(2 k)} \frac{r^{2 k-1 / 2} e^{\mathrm{i} d r^{2} / 2 b}}{b^{2 k}(-\mathrm{i} a / b)^{k+\mathrm{i} \mu}}{ }_{1} F_{1}\left(\begin{array}{c}
k+\mathrm{i} \mu \\
2 k
\end{array} ; \frac{-\mathrm{i} r^{2}}{2 a b}\right) .
\end{aligned}
$$

Needless to say, the joint phases of $-\mathrm{i} a / b$ are needed so as not fall into multivaluation problems. This result, reported in [6], was calculated following the general method to find Mellin transforms of hypergeometric functions due to Majumdar and Basu [4].

Now it is only necessary to perform the Mellin transform of (22), to obtain

$$
{ }^{2} D_{\mu, \mu^{\prime}}^{k}\left(\begin{array}{ll}
a & b \\
c & d
\end{array}\right):=\left({ }^{2} \Phi_{\mu}^{k}, \mathcal{C}\left(\begin{array}{ll}
a & b \\
c & d
\end{array}\right)^{2} \Phi_{\mu^{\prime}}^{k}\right)
$$




$$
\begin{aligned}
= & e^{-\mathrm{i} \pi k} 2^{\mathrm{i}\left(\mu^{\prime}-\mu\right)} \frac{\Gamma(k-\mathrm{i} \mu) \Gamma\left(k+\mathrm{i} \mu^{\prime}\right)}{2 \pi \Gamma(2 k)} \\
& \times b^{-2 k}\left(\frac{-\mathrm{i} d}{b}\right)^{-k+\mathrm{i} \mu}\left(\frac{-\mathrm{i} a}{b}\right)^{-k-\mathrm{i} \mu^{\prime}}{ }_{2} F_{1}\left(\begin{array}{c}
k-\mathrm{i} \mu, k+\mathrm{i} \mu^{\prime} \\
2 k
\end{array} ; \frac{1}{a d}\right) .
\end{aligned}
$$

One should note that the complex power functions are evaluated along the imaginary axis, in the principal sheet where the cut is chosen along the negative real half-axis.

Let us call this the $\mathcal{D}_{k}$-hyperbolic basis (not to confuse it later on with hyperbolic LCTs), because it lies the $\mathcal{D}_{k}^{+}$representation of $\operatorname{Sp}(2, \mathrm{R})$. The corresponding LCT with the integral kernel (23) that transform functions $\mathbf{f}=\{f(\mu)\}_{\mu \in \mathrm{R}} \in \mathcal{L}^{2}(\mathrm{R})$ unitarily, are

$$
\mathbf{f}_{\mathrm{M}} \equiv \mathcal{C}_{\mathrm{M}}: \mathbf{f}={ }^{2} \mathbf{D}^{k} \mathbf{f}, \quad f_{\mathrm{M}}(\mu) \equiv\left(\mathbf{f}_{\mathrm{M}}\right)(\mu)=\int_{-\infty}^{\infty} \mathrm{d} \mu^{\prime 2} D_{\mu, \mu^{\prime}}^{k}(\mathbf{M}) f\left(\mu^{\prime}\right)
$$

Since $J_{1}^{\gamma}$ and $J_{2}^{\gamma}$ are related by similarity through $\mathcal{C} \frac{1}{\sqrt{2}}\left(\begin{array}{cc}1 & -1 \\ 1 & 1\end{array}\right)$ (the square root of the Fourier transform), we obtain an equivalent integral LCT,

$$
{ }^{1} D_{\mu, \mu^{\prime}}^{k}\left(\begin{array}{ll}
a & b \\
c & d
\end{array}\right)={ }^{2} D_{\mu, \mu^{\prime}}^{k} \frac{1}{2}\left(\begin{array}{rr}
a+b+c+d & -a+b-c+d \\
-a-b+c+d & a-b-c+d
\end{array}\right)
$$

In this section we have thus shown three faces of LCTs: the summation kernel (13) for vectors $\left\{f_{n}\right\}_{n \in \mathrm{Z}_{0}^{+}}$, the 'radial' canonical transform integral kernel (18) for functions $\{f(r)\}_{r \in \mathrm{R}^{+}}$and its Hankel transform (21), and the (apparently unknown) $\mathcal{D}_{k}$-hyperbolic transforms (23) and (24) for functions $\{f(\mu)\}_{\mu \in \mathrm{R}}$. These various functions are in fact the coordinates of the same abstract vector $\mathbf{f}$ as $f_{n}={ }_{0}\langle k, k+n \mid \mathbf{f}\rangle, f(r)={ }_{-}\langle k, r \mid \mathbf{f}\rangle, f(\mu)={ }_{1}\langle k, \mu \mid \mathbf{f}\rangle$, etc. In this sense, all faces of the Hilbert space vector $\mathbf{f}$ are related, and so are their LCTs.

\section{The $\mathcal{C}_{s}^{\varepsilon}$ canonical transforms}

Let us now consider the range $\gamma<-\frac{1}{4}$ of the so(2,1) generators in (5), corresponding to centripetal potentials, which also stem from the separation of $D=2$ coordinates in two disjoint patches of hyperbolic coordinates. One patch (indicated by $\sigma=+1$ ) is $x=\rho \cosh \zeta, y=\rho \sinh \zeta$, and the other $(\sigma=-1)$ is $x=\rho \sinh \zeta, y=\rho \cosh \zeta$, for $\rho, \zeta \in \mathrm{R}$. Fourier expansion in $\zeta$ provides $\partial_{\zeta}^{2} \mapsto-\bar{\zeta}^{2}, \bar{\zeta} \in \mathrm{R}$ that yields a centripetal term in the generators (5). In contrast to radial coordinates, which follow the subgroup reduction $\mathrm{Sp}(4, \mathrm{R}) \supset \mathrm{SO}(2) \otimes \mathrm{Sp}(2, \mathrm{R})$, hyperbolic coordinates conform to the reduction $\mathrm{Sp}(4, \mathrm{R}) \supset \mathrm{O}(1,1) \otimes \mathrm{Sp}(2, \mathrm{R})$, where $\mathrm{O}(1,1)$ contains the discrete reflections $\left(\begin{array}{ll}0 & 1 \\ 1 & 0\end{array}\right)$ that interchanges the two values of $\sigma$, and $\left(\begin{array}{cc}1 & 0 \\ 0 & -1\end{array}\right)$ that allows the reduction by even or odd functions in $\rho$, so the range of this hyperbolic radius is reduced to $r=|\rho| \in[0, \infty)$ and $\sigma=\operatorname{sign} \rho$. The Hilbert spaces of functions for the range of representations $\gamma<-\frac{1}{4}$ consists of functions $\_\langle r, \sigma \mid f\rangle \equiv f(r, \sigma) \equiv f_{\sigma}(r)$ whose inner product we can represent with two-vector notation

$$
\mathbf{f}(r) \equiv\left(\begin{array}{l}
f_{+1} \\
f_{-1}
\end{array}\right)(r) \in \mathcal{L}_{2}^{2}\left(\mathrm{R}^{+}\right), \quad(\mathbf{f}, \mathbf{g}):=\sum_{\sigma \in\{+1,-1\}} \int_{0}^{\infty} \mathrm{d} r f_{\sigma}(r)^{*} g_{\sigma}(r),
$$

taking care to note that $\sigma$ is an index of the basis functions $|r, \sigma\rangle_{-}$stemming from the two hyperbolic coordinate patches. When $\gamma=-\frac{1}{4}$, the Bargmann index in (8) is $k=\frac{1}{2}$; for $\gamma<-\frac{1}{4}$, $k$ becomes complex,

$$
k=\frac{1}{2}+\mathrm{i} s, \quad s \in \mathrm{R}, \quad \kappa=\frac{1}{4}+s^{2} \geq \frac{1}{4}
$$


and determines these representations to belong to the Bargmann continuous nonexceptional se$\operatorname{ries} \mathcal{C}_{s}^{\varepsilon}$, where $\varepsilon \in\left\{0, \frac{1}{2}\right\}$ distinguishes between vector and spinor (two-fold cover) representations

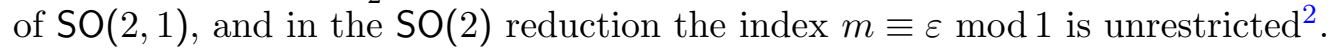

The (reduced) list of eigenfunctions of the generators $J_{X}^{\gamma}$ in (5) is now (cf. (9)),

$$
\begin{aligned}
{ }^{0} \boldsymbol{\Phi}_{m}^{\varepsilon, k}(r)= & \frac{g_{\varepsilon}(k)}{\pi \sqrt{r}}\left(\begin{array}{r}
(-1)^{m-\varepsilon} \sqrt{2 \Gamma(k-m) \Gamma(1-k-m)} W_{m, k-1 / 2}\left(r^{2}\right) \\
2 \Gamma(k+m) \Gamma(1-k+m) W_{-m, k-1 / 2}\left(r^{2}\right)
\end{array}\right), \\
& m+\varepsilon \in \mathrm{Z}, \quad k=\frac{1}{2}+\mathrm{i} s, \quad g_{0}(k)=\cosh \pi s, \quad g_{1 / 2}(k)=\sinh \pi s ; \\
-\boldsymbol{\Phi}_{\rho}^{\varepsilon, k}(r)= & \left(\begin{array}{c}
\delta(\rho-r) \\
0
\end{array}\right) \quad \text { for } \rho \geq 0, \quad \text { and } \quad\left(\begin{array}{c}
0 \\
\delta(|\rho|-r)
\end{array}\right) \quad \text { for } \rho<0 ; \\
{ }^{2} \boldsymbol{\Phi}_{\tau, \mu}^{\varepsilon, k}(r)= & \frac{1}{\sqrt{2 \pi}}\left(\begin{array}{c}
1 \\
\tau
\end{array}\right) r^{-1 / 2+\mathrm{i} \mu}, \quad \mu \in \mathrm{R}, \quad \tau \in\{+1,-1\} ;
\end{aligned}
$$

where $W_{\lambda, \mu}$ is a Whittaker function. The two components of the eigenfunction are distinguished by the non-symplectic matrix $\left(\begin{array}{cc}1 & 0 \\ 0 & -1\end{array}\right) \in \mathrm{O}(1,1)$, which on the algebra generators $(5)$ and $(7)$ can be interpreted as $2 \times 2$ matrix operators $\mathbf{J}_{0}^{\gamma}=\left(\begin{array}{cc}J_{0}^{\gamma} & 0 \\ 0 & -J_{0}^{\gamma}\end{array}\right), \mathbf{J}_{1}^{\gamma}=\left(\begin{array}{cc}J_{1}^{\gamma} & 0 \\ 0 & -J_{1}^{\gamma}\end{array}\right), \mathbf{J}_{2}^{\gamma}=\left(\begin{array}{cc}J_{2}^{\gamma} & 0 \\ 0 & J_{2}^{\gamma}\end{array}\right)$, $\mathbf{J}_{ \pm}^{\gamma}=\left(\begin{array}{cc}J_{ \pm}^{\gamma} & 0 \\ 0 & -J_{ \pm}^{\gamma}\end{array}\right)$.

\subsection{Elliptic basis: discrete $\mathcal{C}_{s}^{\varepsilon}$-LCTs}

The action of the LCT operator $\mathcal{C}_{\mathrm{M}}$ on the discrete eigenfunction basis of $\mathbf{J}_{0}^{\gamma},{ }^{0} \boldsymbol{\Phi}_{m}^{\varepsilon, k}(r)$, for $m-\varepsilon \in \mathbf{Z}$ will be that of an infinite matrix $\mathbf{C}_{\mathrm{M}}^{\varepsilon, k}=\left\|C_{\mathrm{M} ; m, m^{\prime}}^{\varepsilon, k}\right\|$. The transformation of this basis under $\operatorname{Sp}(2, \mathrm{R})$ can be again factorized into the right-ordered product in (10), so that as in (11), we now have

$$
\left(\mathcal{C}\left(\begin{array}{ll}
a & b \\
c & d
\end{array}\right)^{0} \boldsymbol{\Phi}_{m}^{\varepsilon, k}\right)_{\sigma}(r)=\left(\frac{a-\mathrm{i} b}{a+\mathrm{i} b}\right)^{m} \frac{\exp \left(\mathrm{i} \sigma r^{2} \frac{a c+b d}{2\left(a^{2}+b^{2}\right)}\right)}{\left(a^{2}+b^{2}\right)^{1 / 4}}{ }^{0} \boldsymbol{\Phi}_{m, \sigma}^{\varepsilon, k}\left(\frac{r}{\sqrt{a^{2}+b^{2}}}\right),
$$

where the component sign $\sigma$ only appears in the Gaussian exponent. This relation shows that, in the same way as harmonic oscillator functions reproduce under the LCTs (3), and Bessel functions under radial $\mathcal{D}_{k}$ LCTs (16), the Whittaker functions in (26) do likewise under the $\mathcal{C}_{s}^{\varepsilon}$ continuous-series LCTs.

But the following step of finding the LCT matrix elements as in (12), results in a sum of integrals of two Whittaker functions and an oscillating Gaussian, which the authors [6] could not solve. As in (13) this result was obtained before with the traditional $m$-shift operators by Bargmann [2],

$$
\begin{aligned}
& { }^{0} C_{m, m^{\prime}}^{\varepsilon, k}\left(\begin{array}{ll}
a & b \\
c & d
\end{array}\right):=\left({ }^{0} \boldsymbol{\Phi}_{m}^{\varepsilon, k}, \mathcal{C}\left(\begin{array}{ll}
a & b \\
c & d
\end{array}\right){ }^{0} \boldsymbol{\Phi}_{m^{\prime}}^{\varepsilon, k}\right)=\sum_{\sigma} \int_{0}^{\infty} \mathrm{d} r \ldots \\
& \text { for } \stackrel{m \geq m^{\prime}}{=} \frac{2^{2 m^{\prime}}}{m^{\prime} !} \sqrt{\frac{\Gamma(k+m) \Gamma(1-k+m)}{\Gamma(k+m) \Gamma(1-k+m)}} \frac{[(a-d)+\mathrm{i}(b+c)]^{m-m^{\prime}}}{[(a+d)+\mathrm{i}(b-c)]^{m+m^{\prime}}} \\
& \times{ }_{2} F_{1}\left(\begin{array}{c}
k-m^{\prime}, 1-k-m^{\prime} \\
1+m-m^{\prime}
\end{array} ;-\frac{1}{4}\left(a^{2}+b^{2}+c^{2}+d^{2}-2\right)\right), \\
& \text { for } \stackrel{m \leq m^{\prime}}{=}(-1)^{m^{\prime}-m} \frac{2^{2 m}}{m !} \sqrt{\frac{\Gamma\left(k+m^{\prime}\right) \Gamma\left(1-k+m^{\prime}\right)}{\Gamma(k+m) \Gamma(1-k+m)}} \frac{[(a-d)-\mathrm{i}(b+c)]^{m^{\prime}-m}}{[(a+d)+\mathrm{i}(b-c)]^{m^{\prime}+m}}
\end{aligned}
$$

\footnotetext{
${ }^{2}$ I.M. Gel'fand and M.A. Naummark called this the principal representation series [8, 21].
} 


$$
\times{ }_{2} F_{1}\left(\begin{array}{c}
k-m, 1-k-m \\
1+m^{\prime}-m
\end{array} ;-\frac{1}{4}\left(a^{2}+b^{2}+c^{2}+d^{2}-2\right)\right) .
$$

As in (14), but now for infinite vectors of discrete components, $\mathbf{f}=\left\{f_{m}\right\}_{m-\varepsilon \in Z}$, the LCT is

$$
\mathbf{f}_{\mathrm{M}} \equiv \mathcal{C}_{\mathrm{M}}: \mathbf{f}={ }^{0} \mathbf{C}_{\mathrm{M}}^{\varepsilon, k} \mathbf{f}, \quad f_{\mathrm{M} ; m} \equiv\left(\mathbf{f}_{\mathrm{M}}\right)_{m}=\sum_{m^{\prime}=-\infty}^{\infty}{ }^{0} C_{m, m^{\prime}}^{\varepsilon, k}(\mathbf{M}) f_{m^{\prime}}
$$

and is unitary in the Hilbert space of sequences $\ell^{2}(Z)$.

\subsection{Parabolic basis: radial $\mathcal{C}_{s}^{\varepsilon}$-LCTs}

The action of the canonical transform operators $\mathcal{C}_{\mathrm{M}}$ on functions $-\langle\sigma, r \mid f\rangle=f_{\sigma}(r) \in \mathcal{L}_{2}^{2}\left(\mathrm{R}^{+}\right)$will be represented in the continuous series $\mathcal{C}_{s}^{\varepsilon}$ by a $2 \times 2$ matrix of integral kernels

$$
\begin{aligned}
& \mathbf{f}_{\mathrm{M}}(r) \equiv\left(\mathcal{C}_{\mathrm{M}}:\left(\begin{array}{c}
f_{+1} \\
f_{-1}
\end{array}\right)\right)(r)=\int_{0}^{\infty} \mathrm{d} r^{\prime}-\mathbf{C}_{\mathrm{M}}^{\varepsilon, k}\left(r, r^{\prime}\right) \mathbf{f}\left(r^{\prime}\right), \\
& { }^{-} \mathbf{C}_{\mathrm{M}}^{\varepsilon, k}\left(r, r^{\prime}\right)=\left(\begin{array}{ll}
-C_{\mathrm{M} ;+1,+1}^{\varepsilon, k}\left(r, r^{\prime}\right) & -C_{\mathrm{M} ;++1,-1}^{\varepsilon, k}\left(r, r^{\prime}\right) \\
-C_{\mathrm{M} ;-1,+1}^{\varepsilon, k}\left(r, r^{\prime}\right) & -C_{\mathrm{M} ;-1,-1}^{\varepsilon, k}\left(r, r^{\prime}\right)
\end{array}\right) .
\end{aligned}
$$

Here we resort to the reduction $\mathrm{Sp}(4, \mathrm{R}) \supset \mathrm{O}(1,1) \otimes \mathrm{Sp}(2, \mathrm{R})$ as presented in [27], with the precisions made in [6, equations (2.15)] regarding ranges and phases. For the non-exceptional continuous series of representations $\mathcal{C}_{s}^{\varepsilon}$, where $\kappa=k(1-k) \geq \frac{1}{4}$ and

$$
\begin{aligned}
& \varepsilon=0: \quad h_{\varepsilon}=1, \quad k-\frac{1}{2}=\mathrm{i} s, \quad s \geq 0, \\
& \varepsilon=\frac{1}{2}: \quad h_{\varepsilon}=-1, \quad k-\frac{1}{2}=\mathrm{i} s, \quad s>0,
\end{aligned}
$$

where $g_{\varepsilon}(k)$ is given in $(25)$.

The integral kernel elements of $\mathbf{C}_{\mathrm{M}}^{\varepsilon, k}$ are then expressed as

$$
\begin{aligned}
\left({ }^{-} \mathbf{C}_{\mathrm{M}}^{\varepsilon, k}\right)_{\sigma, \sigma^{\prime}}\left(r, r^{\prime}\right)=G_{\mathrm{M} ; \sigma, \sigma^{\prime}}\left(r, r^{\prime}\right) H_{\sigma, \sigma^{\prime}}^{\varepsilon, k}\left(-r r^{\prime} / b\right), & \\
G_{\mathrm{M} ; \sigma, \sigma^{\prime}}\left(r, r^{\prime}\right) & :=\frac{\sqrt{r r^{\prime}}}{2 \pi|b|} \exp \left(\mathrm{i} \frac{d \sigma r^{2}+a \sigma^{\prime} r^{\prime 2}}{2 b}\right), \\
H_{+1,+1}^{\varepsilon, k}(\zeta) & :=\mathrm{i} \pi\left(e^{-\pi s} H_{2 \mathrm{i} s}^{(1)}\left(\zeta+\mathrm{i} 0^{+}\right)-h_{\varepsilon} e^{\pi s} H_{2 \mathrm{i} s}^{(2)}\left(\zeta-\mathrm{i} 0^{+}\right)\right) \\
& =h_{\varepsilon} H_{-1,-1}^{\varepsilon, k}(\zeta)=h_{\varepsilon} H_{+1,+1}^{\varepsilon, k}(-\zeta)=H_{+1,+1}^{\varepsilon, 1-k}(\zeta), \\
H_{+1,-1}^{\varepsilon, k}(\zeta) & :=4(-\operatorname{sign} \zeta) g_{\varepsilon}(k) K_{2 \mathrm{i} s}(|\zeta|) \\
& =h_{\varepsilon} H_{-1,+1}^{\varepsilon, k}(\zeta)=h_{\varepsilon} H_{+1,-1}^{\varepsilon, k}(-\zeta)=h_{\varepsilon} H_{+1,-1}^{\varepsilon, 1-k}(\zeta),
\end{aligned}
$$

where $H_{\nu}^{(1)}$ and $H_{\nu}^{(2)}$ are the Hankel functions of the first and second kind valued above and below the branch cut, $K_{\nu}$ is the Macdonald function, while $g_{\varepsilon}(k)$ and $h_{\varepsilon}(k)$ are given in (28).

The $2 \times 2$ kernel LCT in (27), $C_{\mathrm{M} ; \sigma, \sigma^{\prime}}^{\varepsilon, k}\left(r, r^{\prime}\right), r, r^{\prime} \in \mathrm{R}^{+}$, can be written also as $C_{\mathrm{M}}^{\varepsilon, k}\left(\rho, \rho^{\prime}\right)$, with $\rho:=\sigma r \in \mathrm{R}$. In that case, the kernel $\mathcal{C}_{s}^{\varepsilon}$ has been expressed [6] in terms of confluent hypergeometric functions,

$$
\begin{aligned}
C_{\mathrm{M}}^{\varepsilon, k}\left(\rho, \rho^{\prime}\right)= & \frac{(\operatorname{sign} b)^{2 \varepsilon} h_{\varepsilon}^{\left(1+\operatorname{sign} \rho^{\prime}\right) / 2} g_{\varepsilon}(k)}{\pi|b|} \exp \left(\mathrm{i} \frac{d \sigma \rho^{2}-2 \eta \rho \rho^{\prime}+a \sigma^{\prime} \rho^{\prime 2}}{2 b}\right) \sqrt{\rho \rho^{\prime}} \\
& \times\left\{\left[\Gamma(1-2 k)\left|\frac{\rho \rho^{\prime}}{2 b}\right|^{2 k-1}{ }_{1} F_{1}\left(\begin{array}{c}
2 k-\frac{1}{2} \\
4 k-1
\end{array} ; \frac{2 \mathrm{i} \rho \rho^{\prime}}{\eta b}\right)\right]+[k \leftrightarrow 1-k]\right\},
\end{aligned}
$$


where $\sigma=\operatorname{sign} \rho, \sigma^{\prime}=\operatorname{sign} \rho^{\prime} ; \eta=1$ for $\sigma=\sigma^{\prime}$ and $\eta=-\mathrm{i}$ for $\sigma \neq \sigma^{\prime}$. In particular, for $b=0$,

$$
\lim _{b \rightarrow 0}{ }^{-} C_{\mathrm{M}}^{\varepsilon, k}\left(\rho, \rho^{\prime}\right)=\frac{(\operatorname{sign} a)^{2 k}}{\sqrt{|a|}} \exp \left(\operatorname{i} \operatorname{sign} \rho \frac{c \rho^{2}}{2 a}\right) \delta\left(\rho^{\prime}-\rho /|a|\right) .
$$

We are not addressing the Bargmann exceptional representation series, for which in (28) one has $k=\frac{1}{2}+s$, with $0<s<\frac{1}{2}$ overlapping the exceptional interval, and for which (29)-(32) appears to be a valid integral transform. Treatment of the exceptional continuous series can be seen in [5].

\subsection{Hyperbolic basis and their $\mathcal{C}_{s}^{\varepsilon}$-LCTs}

The eigenfunctions ${ }^{2} \boldsymbol{\Phi}_{\tau, \mu}^{\varepsilon, k}(r)$ of $J_{2}^{\gamma}$ in the continuous series representations form a generalized basis for a Hilbert space $\mathcal{L}_{2}^{2}(\mathrm{R})$, and are given in (25) with $\tau \in\{+1,-1\}, \mu \in \mathrm{R}$ and $r \in \mathrm{R}^{+}$. They can provide an LCT form with a $2 \times 2$ matrix of integral kernels that are the double Mellin transforms of the LCT kernel in the position basis given by (29)-(32) or (33). Using the technique of Basu and Majumdar on the latter, [6] reports

$$
\begin{aligned}
{ }^{2} C_{\tau, \mu ; \tau^{\prime}, \mu^{\prime}}^{\varepsilon, k}\left(\begin{array}{ll}
a & b \\
c & d
\end{array}\right):= & \left({ }^{2} \Phi_{\tau, \mu}^{\varepsilon, k}, \mathcal{C}\left(\begin{array}{ll}
a & b \\
c & d
\end{array}\right){ }^{2} \Phi_{\tau^{\prime}, \mu^{\prime}}^{\varepsilon, k}\right) \\
= & \frac{(-\operatorname{sign} b)^{2 \varepsilon} g_{\varepsilon}(k)}{2 \pi}\left[\left(\alpha_{k}+\frac{\tau \tau^{\prime} h_{\varepsilon}}{\alpha_{k}}+\tau^{\prime} \beta_{k}+\frac{\tau h_{\varepsilon}}{\beta_{k}}\right) T_{k}\right. \\
& \left.+\left(h_{\varepsilon} \alpha_{1-k}+\frac{\tau \tau^{\prime}}{\alpha_{1-k}}+\tau^{\prime} \beta_{1-k}+\frac{\tau h_{\varepsilon}}{\beta_{1-k}}\right) T_{1-k}\right]
\end{aligned}
$$

where

$$
\begin{aligned}
& T_{k}:=\frac{\Gamma(1-2 k) \Gamma(k-\mathrm{i} \mu) \Gamma\left(k+\mathrm{i} \mu^{\prime}\right)}{|a|^{k-\mathrm{i} \mu^{\prime}}|b|^{\mathrm{i}\left(\mu-\mu^{\prime}\right)}|d|^{k-\mathrm{i} \mu}}{ }_{2} F_{1}\left(\begin{array}{c}
k-\mathrm{i} \mu, k+\mathrm{i} \mu^{\prime} \\
2 k
\end{array} ; \frac{1}{a d}\right), \\
& \alpha_{k}:=\exp \left(\mathrm{i} \frac{1}{2} \pi\left[\left(k+\mathrm{i} \mu^{\prime}\right) \operatorname{sign} a b+(k-\mathrm{i} \mu) \operatorname{sign} b d\right]\right), \\
& \beta_{k}:=\exp \left(\mathrm{i} \frac{1}{2} \pi\left[-\left(k+\mathrm{i} \mu^{\prime}\right) \operatorname{sign} a b+(k-\mathrm{i} \mu) \operatorname{sign} b d\right]\right),
\end{aligned}
$$

and where $g_{\varepsilon}(k)$ and $h_{\varepsilon}$ are given in (28).

Note that the two components of this $\mathcal{L}_{2}^{2}(\mathrm{R})$ are distinct from the two components of the Hilbert space $\mathcal{L}_{2}^{2}\left(\mathrm{R}^{+}\right)$in the parabolic case of the previous subsection. In this hyperbolic $\mathcal{C}_{s}^{\varepsilon}$ representation, LCTs are maps of two-component functions by the integral

$$
\begin{aligned}
& \mathbf{f}_{\mathrm{M}} \equiv \mathcal{C}_{\mathrm{M}}: \mathbf{f}={ }^{2} \mathbf{C}^{\varepsilon, k} \mathbf{f}, \\
& f_{\mathrm{M} ; \tau}(\mu) \equiv\left(\mathbf{f}_{\mathrm{M}}\right)_{\tau}(\mu)=\sum_{\tau^{\prime} \in\{-1,1\}} \int_{-\infty}^{\infty} \mathrm{d} \mu^{\prime 2} C_{\tau, \mu ; \tau^{\prime} \mu^{\prime}}^{\varepsilon, k}(\mathbf{M}) f_{\tau^{\prime}}\left(\mu^{\prime}\right)
\end{aligned}
$$

and is unitary in that Hilbert space.

\section{Concluding remarks}

The recompilation of the six forms that LCTs have in the elliptic, parabolic and hyperbolic subgroup bases of $\operatorname{Sp}(2, \mathrm{R})$ in the $\mathcal{D}_{k}$ and $\mathcal{C}_{s}^{\varepsilon}$ representation series, has been made for the purpose of placing the better-known LCT forms, i.e., the 'linear' (3), the 'radial' (17), and the (lesser known) 'hyperbolic' (29)-(32), in the general context of group representation theory. We still have to justify the appellative of canonical though, because it is a term associated with the preservation of area elements in classical phase space, the conservation of energy in paraxial wave optics, and of uncertainty in quantum mechanics. 
Classically, the $2 \times 2$ symplectic matrix $\mathbf{M}=\left(\begin{array}{ll}a & b \\ c & d\end{array}\right)$ in (1) acts on the phase-space coordinates, written $(r, p)^{\top}$ as if they were a 2 -vector. The correspondence (2) carries its action as a $(2+1)$ Lorentz transformation of the 3 -vector of phase space functions

$$
\left(\xi_{0}, \xi_{1}, \xi_{2}\right)^{\top}:=\left(\frac{1}{4}\left(p^{2}+\gamma / r^{2}+r^{2}\right), \frac{1}{4}\left(p^{2}+\gamma / r^{2}-r^{2}\right), \frac{1}{2} r p\right)^{\top},
$$

which close under Poisson brackets into the same algebra, with $\mathrm{i}[\cdot, \cdot] \mapsto\{\cdot, \cdot\}$, as the operators $\left\{J_{i}^{\gamma}\right\}_{i=0}^{2}$ in (6),

$$
\left\{\xi_{1}, \xi_{2}\right\}=-\xi_{0}, \quad\left\{\xi_{2}, \xi_{0}\right\}=\xi_{1}, \quad\left\{\xi_{0}, \xi_{1}\right\}=\xi_{2} .
$$

We may consider these relations as the Berezin brackets defining the Lorentz algebra. The square length of the vector (34) is $\xi_{1}^{2}+\xi_{2}^{2}-\xi_{0}^{2}=-\frac{1}{4} \gamma$ [instead of $-\frac{1}{4} \gamma+\frac{3}{16}$ in the operator case (8)], and this surface is conserved under Lorentz transformations. For centrifugal $\gamma>0$ or centripetal $\gamma<0$ these surfaces are respectively a two-sheeted or one-sheeted hyperboloid, and a cone for $\gamma=0$; these surfaces are also symplectic manifolds. Since classically $r= \pm \sqrt{2\left(\xi_{0}-\xi_{1}\right)}$ and $p=2 \xi_{2} / r$, a linear transformation in the $\xi$ 's is nonlinear in $(r, p)^{\top}$, Moshinsky termed these nonlinear canonical transformations [14, 18].

We count the $\operatorname{Sp}(2, R)$ canonical transforms collected here as different faces of LCTs acting on different function Hilbert spaces: $\ell^{2}\left(Z_{0}^{+}\right)$and $\ell^{2}(Z)$ in the elliptic basis of $\mathcal{D}_{k}^{+}$and $\mathcal{C}_{s}^{\varepsilon}$ representations respectively; similarly, $\mathcal{L}^{2}\left(\mathrm{R}^{+}\right)$and $\mathcal{L}_{2}^{2}\left(\mathrm{R}^{+}\right)$in the parabolic basis, and $\mathcal{L}^{2}(\mathrm{R})$ and $\mathcal{L}_{2}^{2}(\mathrm{R})$ in the hyperbolic basis. Of course, the best known ones are those in the eigenbasis of $J_{-}^{\gamma}=\frac{1}{2} r^{2}$ understood as the position observable, which include the 'radial' LCTs in the $\mathcal{D}_{k}^{+}$, particularly the 'linear' transform kernels written by Collins [7] and Moshinsky et al. [17], which are genuinely fit to describe the signals or wavefunctions that traverse a paraxial optical or quantum mechanical system. For the same parabolic subgroup, the 'hyperbolic' LCTs in the $\mathcal{C}_{s}^{\varepsilon}$ representations have found no proper application, save their use in [6] to find all $S L(2, R)$ group representations in all subgroup and mixed bases reported there. Widely used in other contexts, the Bargmann results of 1947 [2] that provide the matrix representations of the Lorentz group, uses the elliptic subgroup to provide the row indices. This has been used in [1] to propose a covariant discretization for axis-symmetric $D=2$ systems, where the limit of the discrete to the continuous radial model is pointedly addressed. Among the six LCT forms presented above, similar limits from elliptic or hyperbolic to parabolic subgroup bases should occur in all representations. Representations referred to the hyperbolic subgroup were investigated by Mukunda and Radhakrishnan [20] but have also failed to be associated with some model of physical system.

Uncertainty relations are preserved under all LCTs, but they stem from so $(2,1)$ - not from the Heisenberg-Weyl algebra - and involve the coordinate $\frac{1}{2} r^{2}>0$. Recall that the mean of a selfadjoint operator $J$ in a wavefield $\psi$ is $\bar{\jmath}_{\psi}:=(\psi, J \psi)$, with the inner product appropriate to its Hilbert space; its dispersion is then $\Delta_{\psi}(J):=\left\|\left(J-\bar{\jmath}_{\psi}\right) \psi\right\|^{2}$. Note also that from the commutator between $J_{-}=\frac{1}{2} r^{2}$ and its (Fourier) $\mathcal{C}\left(\begin{array}{cc}0 & -1 \\ 1 & 0\end{array}\right)$ transform operator $J_{+}$, is $\left[J_{-}, J_{+}\right]=2 \mathrm{i} J_{2}$, and it follows that

$$
\Delta_{\psi}\left(J_{-}\right) \Delta_{\psi}\left(J_{+}\right)=\Delta_{\psi}\left(J_{-}\right) \Delta_{\widetilde{\psi}}\left(J_{-}\right) \geq \frac{1}{4}\left|\left(\psi, J_{2} \psi\right)\right|^{2},
$$

where $\widetilde{\psi}=\mathcal{C}\left(\begin{array}{cc}0 & -1 \\ 1 & 0\end{array}\right) \psi$. In the special case of the original LCT (3) identified by (20), this can be written in the form of a Robertson uncertainty relation $\left\langle r^{2}\right\rangle_{\psi}\left\langle p^{2}\right\rangle_{\psi} \geq \frac{1}{4}\langle(r p+p r)\rangle_{\psi}^{2}$ [24].

We have not addressed in any depth the exceptional interval $0<k<1$, i.e., $-\frac{1}{4} \leq \gamma<\frac{3}{4}$ characterizing weak centripetal and centrifugal potentials, which Bargmann treated with a nonlocal measure $[2,5]$. Also there, the discrete and exceptional series overlap, and its generators $J_{X}^{\gamma}$ have one-parameter families of self-adjoint extensions [30], with the result that their spectra are 
generally not equally spaced, save for the Friedricks extension in the $\mathcal{D}_{k}$ representation series. The various limits to the point $k=\frac{1}{2}$ are particularly troublesome [6].

Perhaps most important is the computer use of LCTs to model physical systems, pursued since the 1990s, in a quest that is running into hundreds of references [3, 12, 13, 22] where finite data sets or pixellated images are subject to LCT matrices of $N \times N$. Of course, we know that noncompact groups such as $\mathrm{Sp}(2, \mathrm{R})$ cannot have finite-dimensional unitary representations. So, approximations have to be made, either through sampling functions and kernels, or using the finite-dimensional $k<0$ representations of the $\mathcal{D}_{k}$ series mentioned in Section 2; in both strategies the matrices will not be unitary, but only in the second will they faithfully represent the LCT group $\mathrm{Sp}(2, \mathrm{R})$. Perhaps the best approximation strategy is to use a truncated elliptic basis $\left\{{ }^{0} \Phi_{m}^{k}(r)\right\}_{m=k}^{k+N-1}$ of functions that are radial oscillator modes; the matrices would by neither unitary nor would faithfully represent the group; but if the signal contains mostly low energy modes it could be fairly approximated by the first $N$ modes, and with an inherent $N \rightarrow \infty$ limit to a representation that is both unitary and faithful.

\section{Acknowledgements}

The Symposium on Superintegrability, Exact Solvability and Special Functions (Cuernavaca, 2024 February 2012) was supported by the Centro Internacional de Ciencias AC, Fondo "Alfonso Nápoles Gándara", Instituto de Ciencias Físicas, Instituto de Matemáticas, and Intercambio Académico of the Coordinación de la Investigación Científica, Universidad Nacional Autónoma de México. This work was supported by the Óptica Matemática projects PAPIIT-UNAM 101011 and SEP-CONACyT 79899.

\section{References}

[1] Atakishiyev N.M., Nagiyev S.M., Vicent L.E., Wolf K.B., Covariant discretization of axis-symmetric linear optical systems, J. Opt. Soc. Amer. A 17 (2000), 2301-2314.

[2] Bargmann V., Irreducible unitary representations of the Lorentz group, Ann. of Math. (2) 48 (1947), 568640.

[3] Barker L., Candan Ç., Hakioğlu T., Kutay M.A., Ozaktas H.M., The discrete harmonic oscillator, Harper's equation, and the discrete fractional Fourier transform, J. Phys. A: Math. Gen. 33 (2000), 2209-2222.

[4] Basu D., The Lorentz group in the oscillator realization. I. The group $\mathrm{SO}(2,1)$ and the transformation matrices connecting the $\mathrm{SO}(2)$ and $\mathrm{SO}(1,1)$ bases, J. Math. Phys. 19 (1978), 1667-1670.

[5] Basu D., Bhattacharyya T., The Gel'fand realization and the exceptional representations of SL $(2, \mathbf{R})$, J. Math. Phys. 26 (1985), 12-17.

[6] Basu D., Wolf K.B., The unitary irreducible representations of $\operatorname{SL}(2, \mathbf{R})$ in all subgroup reductions, J. Math. Phys. 23 (1982), 189-205.

[7] Collins S.A.J., Lens-system diffraction integral written in terms of matrix optics, J. Opt. Soc. Amer. A 60 (1970), 1168-1177.

[8] Gel'fand I.M., Naŭmark M.A., Unitary representations of the Lorentz group, Izvestiya Akad. Nauk SSSR. Ser. Mat. 11 (1947), 411-504.

[9] Gilmore R., Lie groups, Lie algebras, and some of their applications, John Wiley \& Sons, New York, 1974.

[10] Gradshteyn I.S., Ryzhik I.M., Table of integrals, series, and products, sixth ed., Academic Press Inc., San Diego, CA, 2000.

[11] Han D., Kim Y.S., Noz M.E., Wigner rotations and Iwasawa decompositions in polarization optics, Phys. Rev. E 60 (1999), 1036-1041, quant-ph/0408181.

[12] Healy J.J., Sheridan J.T., Fast linear canonical transforms, J. Opt. Soc. Amer. A 27 (2010), 21-30.

[13] Healy J.J., Sheridan J.T., Sampling and discretization of the linear canonical transform, Signal Process. 89 (2009), 641-648. 
[14] Mello P.A., Moshinsky M., Nonlinear canonical transformations and their representations in quantum mechanics, J. Math. Phys. 16 (1975), 2017-2028.

[15] Monzón J.J., Sánchez-Soto L.L., Multilayer optics as an analog computer for testing special relativity, Phys. Lett. A 262 (1999), 18-26.

[16] Moshinsky M., Quesne C., Linear canonical transformations and their unitary representations, J. Math. Phys. 12 (1971), 1772-1780.

[17] Moshinsky M., Quesne C., Oscillator systems, in Proceedings of the 15th Solvay Conference in Physics (1970), Gordon and Breach, New York, 1974, 233-257.

[18] Moshinsky M., Seligman T.H., Wolf K.B., Canonical transformations and the radical oscillator and Coulomb problems, J. Math. Phys. 13 (1972), 901-907.

[19] Mukunda N., Aravind P.K., Simon R., Wigner rotations, Bargmann invariants and geometric phases, J. Phys. A: Math. Gen. 36 (2003), 2347-2370.

[20] Mukunda N., Radhakrishnan B., New forms for the representations of the three-dimensional Lorentz group, J. Math. Phys. 14 (1973), 254-258.

[21] Naŭmark M.A., Linear representations of the Lorentz group, The Macmillan Co., New York, 1964.

[22] Pei S.C., Ding J.J., Closed-form discrete fractional and affine Fourier transforms, IEEE Trans. Signal Process. 48 (2000), 1338-1353.

[23] Quesne C., Moshinsky M., Canonical transformations and matrix elements, J. Math. Phys. 12 (1971), 1780-1783.

[24] Robertson H.P., The uncertainty principle, Phys. Rev. 34 (1929), 163-264.

[25] Wolf K.B., Canonical transforms. I. Complex linear transforms, J. Math. Phys. 15 (1974), 1295-1301.

[26] Wolf K.B., Canonical transforms. II. Complex radial transforms, J. Math. Phys. 15 (1974), 2102-2111.

[27] Wolf K.B., Canonical transforms. IV. Hyperbolic transforms: continuous series of SL(2, R) representations, J. Math. Phys. 21 (1980), 680-688.

[28] Wolf K.B., Geometric optics on phase space, Texts and Monographs in Physics, Springer-Verlag, Berlin, 2004.

[29] Wolf K.B., Integral transforms in science and engineering, Mathematical Concepts and Methods in Science and Engineering, Vol. 11, Plenum Press, New York, 1979.

[30] Wolf K.B., Aceves-de-la Cruz F., Dependence of $s$-waves on continuous dimension: the quantum oscillator and free systems, Fortschr. Phys. 54 (2006), 1083-1108.

[31] Yonte T., Monzón J.J., Sánchez-Soto L.L., Cariñena J.F., López-Lacasta C., Understanding multilayers from a geometrical viewpoint, J. Opt. Soc. Amer. A 19 (2002), 603-609, physics/0104050. 